
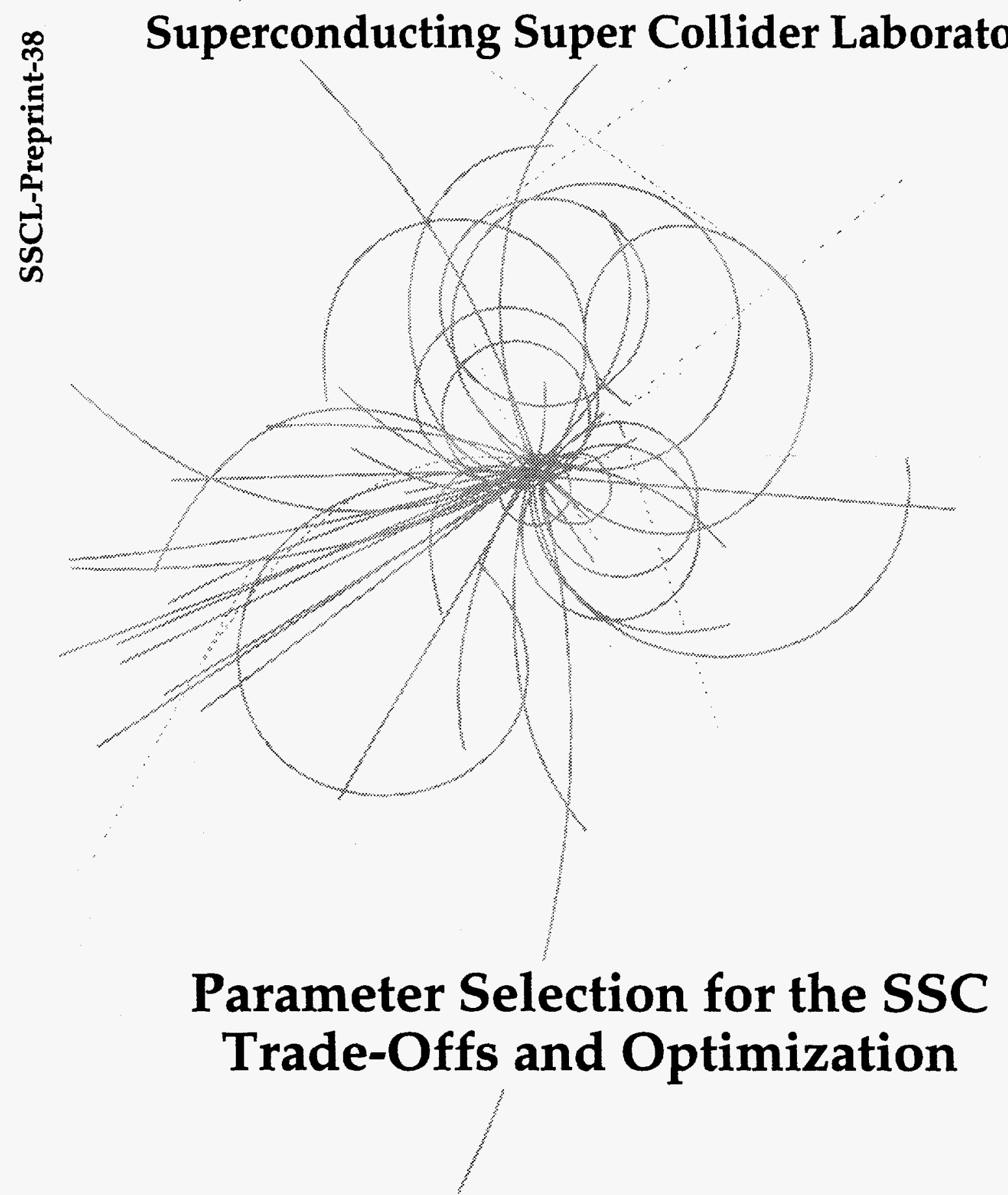

D. Edwards and M. Syphers

October 1991 


\title{
Parameter Selection for the SSC Trade-Offs and Optimization
}

\author{
D. Edwards and M. Syphers \\ Superconducting Super Collider Laboratory* \\ 2550 Beckleymeade Avenue \\ Dallas, TX 75237
}

October 1991

\section{DISCLAIMER}

This report was prepared as an account of work sponsored by an agency of the United States Government. Neither the United States Government nor any agency thereof, nor any of their employees, makes any warranty, express or implied, or assumes any legal liability or responsibility for the accuracy, completeness, or usefulness of any information, apparatus, product, or process disclosed, or represents that its use would not infringe privately owned rights. Reference herein to any specific commercial product, process, or service by trade name, trademark, manufacturer, or otherwise does not necessarily constitute or imply its endorsement, recommendation, or favoring by the United States Government or any agency thereof. The views and opinions of authors expressed herein do not necessarily state or reflect those of the United States Government or any agency thereof.

" Operated by the Universities Research Association, Inc., for the U.S. Department of Energy under Contract No. DE-AC35-89ER40486. 


\section{DISCLAIMER}

Portions of this document may be illegible in electronic image products. Images are produced from the best available original document. 


\title{
Parameter Selection for the SSC Trade-Offs and Optimization
}

\author{
D. A. Edwards and M. J. Syphers \\ SSC Laboratory* \\ Dallas TX 75237
}

October 14, 1991

\begin{abstract}
In November of 1988 , a site was selected in the state of Texas for the SSC. In January of 1989, the SSC Laboratory was established in Texas to adapt the design of the collider to the site and to manage the construction of the project. This paper describes the evolution of the SSC design since site selection, notes the increased concentration on the injector system, and addresses the rationale for choice of parameters.
\end{abstract}

\section{Introduction}

The basic parameters of the SSC shown in Table 1 were established, for the most part, some eight years ago. In the summer of 1984, a Central Design

Table 1: SSC Parameters

\begin{tabular}{|l|c|l|}
\hline Particle Species & Proton & \\
\hline Energy of Each Beam & 20 & $\mathrm{TeV}$ \\
\hline Peak Luminosity & $10^{33} \mathrm{~cm}^{2} \mathrm{sec}$ & \\
\hline No. of Interaction Regions & up to 4 initially & \\
\hline Storage Time (typical) & 20 & hours \\
\hline Test Beam Energy & 200 & $\mathrm{GeV}$ \\
\hline Availability & 80 & $\%$ \\
\hline
\end{tabular}

Group (CDG) was established at Lawrence Berkeley Laboratory to organize the

\footnotetext{
* Operated by the Universities Research Association, Inc., for the U. S. Department of Energy.
} 
Table 2: SSC Injector Parameters

\begin{tabular}{|c|c|c|c|c|c|}
\hline & Collider & HEB & MEB & LEB & Linac \\
\hline Energy & $20 \mathrm{TeV}$ & $2 \mathrm{TeV}$ & $200 \mathrm{GeV}$ & $12 \mathrm{GeV}$ & $600 \mathrm{MeV}$ (K.E.) \\
\hline Circumference (m) & 87120 & 10800 & 3960 & 570 & \\
\hline Superconducting/Normal & $\overline{\mathrm{SC}}$ & $\mathrm{SC}$ & $\mathrm{N}$ & $\mathbf{N}$ & $\mathrm{N}$ \\
\hline Bunch Spacing & 5 & 5 & 5 & 5 & \\
\hline Emittance (rms,norm., $10^{-6} \mathrm{~m}$ ) & $1.0 \pi$ & $0.8 \pi$ & $0.7 \pi$ & $0.6 \pi$ & $<0.4 \pi$ \\
\hline Protons/bunch $\left(10^{10}\right)$ & 0.75 & 1 & 1 & 1 & \\
\hline Cycle Time & & $4.5 \mathrm{~min}$. & $4.5 \mathrm{sec}$. & $0.1 \mathrm{sec}$ & \\
\hline
\end{tabular}

SSC design effort. Under the leadership of Maury Tigner, the CDG developed a sound design for a generic version of the SSC independent of site, yet more importantly, developed a design procedure that could be readily modified to the conditions of the selected site.

At the SSC Laboratory, effort has concentrated on tailoring the generic design to the Texas site and fleshing out the injector specifications. (See Table 2.) Additionally, SSC Laboratory personnel have felt that some steps in the direction of design conservatism should be taken. These will be described below. In the following sections we take up the various accelerators in order of descending energy.

\section{Collider Parameters}

Injection into the Collider is the dominant factor in determining the Collider parameters. Since 16 cycles of the High Energy Booster (HEB), each of four minute duration, are required to fill the Collider, particles must be able to endure this environment for $10^{7}$ revolutions about the accelerator without degradation. The present Collider design parameters are shown in Table 3 tugether with the 1986 CDR [1] values.

\subsection{Motivations for Change}

Accelerator experiments performed at Fermilab and at CERN revealed long-term particle beam loss mechanisms in the presence of guide fields with a significant admixture of nonlinearity. These observations were one of the early warning signs leading to the question of long-term stability in the SSC at injection. The causes and mechanisms of the observed diffusion are still not completely understood. 
Concurrently, accelerator studies took place using the Fermilab Main Ring where the injection environment is such that the natural dynamic aperture is on the order of the emittance of the incoming beam from the Booster synchrotron $[2,3]$. The measured geometrical admittance of the Main Ring is more than three times the equilibrium emittance arrived at by dynamical processes. After 20 years of operation the beam behavior in the Main Ring is not adequately understood. Though the Main Ring was not designed as a storage ring, experience with this synchrotron prompts one to look even more closely at the particle dynamics associated with the SSC at its injection energy.

Complementary to the experiments mentioned above were numerical simulations. A striking feature of these simulations was that in order to account, even qualitatively, for the results a rather detailed model of the synchrotron had to be developed and the tracking had to continue for orders of magnitude more turns than previously expected. Essential ingredients of the model were synchrotron oscillations, random and systematic multipole magnetic field errors, and closed orbit distortions. These results led to an extensive study of the long term behavior of particles at the injection conditions of the Collider. When this environment was first simulated for the CDR design, the resulting data indicated a dynamic aperture for $10^{5}$ turns of only $5 \mathrm{~mm}$. Simply extrapolation of the data suggests that the dynamic aperture for a particle to survive $10^{7}$ turns would be on the order of one millimeter. Though this might be a slight underestimate, nevertheless it was hard to feel comfortable with the long-term dynamic aperture of this accelerator.

Table 3: Collider Parameters

\begin{tabular}{|l|c|c|l|}
\hline & Present & $1986 \mathrm{CDR}$ & \\
\hline Injection Energy & 2.0 & 1.0 & $\mathrm{TeV}$ \\
\hline Circumference & 87120 & 82944 & $\mathrm{~m}$ \\
\hline Cell length & 90 & 96 & $\mathrm{~m}$ \\
\hline Cell phase advance & 90 & 60 & $\mathrm{deg}$ \\
\hline Dipole Coil Diameter & 50 & 40 & $\mathrm{~mm}$ \\
\hline Dipole Field & 6.60 & 6.60 & $\mathrm{~T}$ \\
\hline Max. $\beta$ in Arc & 305 & 332 & $\mathrm{~m}$ \\
\hline Max. dispersion in Arc & 1.8 & 3.9 & $\mathrm{~m}$ \\
\hline Beam-beam $\Delta \nu$ (H.O.) & & & \\
\hline Beam-beam $\Delta \nu$ (L.R.) & & & \\
\hline$\beta^{*}$ & 0.5 & 0.5 & $\mathrm{~m}$ \\
\hline Crossing angle & $<150$ & $<150$ & $\mu \mathrm{rad}$ \\
\hline
\end{tabular}




\subsection{Analytical Input}

To study the parameter space of the Collider design, one needs to see the "handles" available. The three major accelerator parameters explored have been the quadrupole spacing in the arcs, the injection energy, and the coil diameter of the superconducting dipole magnets [4].

The first order effect that the quadrupole spacing has on the accelerator performance is the determination of the beam size. For a quadrupole spacing (half cell length) $L$ and a betatron phase advance of $90^{\circ}$ per cell, the maximum amplitude function of the cell is $\hat{\beta}=3.41 \mathrm{~L}$. The dispersion function varies as the square of the quadrupole spacing and $\hat{D}=2.71 L^{2} / R$, where $R$ is the average radius of the arc. The maximum rms beam size within a cell is then

$$
\hat{\sigma}=\sqrt{\frac{\hat{\beta} \epsilon_{N}}{\pi \gamma}+\hat{D}^{2}\left(\frac{\sigma_{p}}{p}\right)^{2}}
$$

where $\epsilon_{N}$ is the rms, normalized emittance, $\gamma$ is the ratio of beam energy to the proton rest energy, and $\sigma_{p} / p$ is the rms momentum spread of the beam.

In addition to the physical extent of the beam being smaller, a shorter half cell length can result in an increase in the linear aperture. For example, the chromaticity due to systematic sextupole errors varies as $L^{3}$. The higher order multipoles have even higher dependences on half-cell length; for instance, the tune shift of off-momentum particles due to decapole errors $\left(b_{4}\right)$ varies as the quadrupole spacing to the seventh power.

Due to the adiabatic damping of betatron oscillations as the energy of the beam is raised, a higher injection energy for the Collider rings implies a smaller beam size and hence reduced aperture requirements. More importantly, the "persistent" current multipoles at injection will be lessened. By doubling the injection energy from $1 \mathrm{TeV}$ to $2 \mathrm{TeV}$ the sextupole coefficient is cut by over a factor of two and coupled with the cell length change indicated in Table 3 , the chromaticity of the Collider ring due to persistent current $b_{2}$ is reduced to 700 units from 3400 units. It also relaxes the alignment requirements of the dipoles enough to eliminate the need for bore tube correctors. In addition, the betatron oscillation amplitudes of the beam particles are reduced by $30 \%$ due to adiabatic damping and, in conjunction with the new half cell spacing, the beam size is reduced by $50 \%$.

The good field region of the Collider magnets can be increased by increasing the magnet coil diameter, keeping the field shape the same. If the transverse dimensions of the magnet (indicated by the coil radius $R_{o}$ ) are increased, then $b_{n} \sim 1 / R_{o}^{n}$. This scaling law applies to the systematic, or average, multipoles in the magnets which are generated by the magnet geometry or by persistent currents.

If coil placement errors are independent of the scale of the magnet, then the rms values of the multipoles will scale like $\sigma_{b_{n}} \sim 1 / R_{o}^{n+1}$. On the other hand, if 
placement errors scale in proportion to the size of the magnet, then $\sigma_{b_{n}} \sim 1 / R_{o}^{n}$. In either case the spread in the higher order multipoles are significantly reduced by increasing the coil radius. For comparison studies, the geometric mean of these two cases was used to perform the scaling of random multipoles, i.e., $\sigma_{b_{n}} \sim$ $1 / R_{o}^{n+1 / 2}$.

While many effects of various design parameters on beam performance at injection can be estimated analytically, verification with long-term particle tracking is still necessary. In particular, tracking is necessary for the unraveling of magnet coil diameter dependencies as well as quantitative verification of effects of the other two changes.

\subsection{Simulations and their Parameterization}

In the Collider, beam tracking results of particle loss for a model based on standard cells $[5,6]$ have been systematically analyzed in order to obtain a method of comparison from one set of tracking plots to another [7] and to reduce statistical and conceptual biases. The results then lend themselves to straightforward empirical parameterization, leading to functional relationships between aperture and number of turns to loss, with, as examples, bend and quad coil diameters, at varoius momentum, off set injection energy, cell length, on phase advance per cell as parameters. An attempt will be made to tie this parametric information to approximate cost differentials in order to gain general insight into whether or not the design is reasonably cost optimized. Similar effort is underway for the High Energy Booster injection situation, and is discussed later in this paper.

The survival plots have been analyzed to find the mean amplitude for loss and its standard deviation as a function of the number of turns. The "aperture" is then defined as the point two standard deviations within the mean. Both the mean and the standard deviation are fit as a function of the number of turns, $n$, and extrapolated to $n=10^{7}$ to define the " $10^{7} \mathrm{n}$ aperture."

The expressions used are

$$
a(n)=B n^{-0.065}
$$

where $a$ is the mean amplitude, and $B$ is to be found for each set of data. For the standard deviation, the empirical relation is

$$
d a(n)=1.8 n^{-0.065} m m
$$

Then the aperture, $A$, is given by

$$
\begin{aligned}
A(n) & =B n^{-0.065}-2 \cdot 1.8 m m n^{-0.065} \\
& =(B-3.6 m m) n^{-0.065}
\end{aligned}
$$


As the results of the analysis are given at $n=10^{7}$ and $\delta p / p \approx 5 \times 10^{-4}\left(5 \sigma_{p} / p\right)$, the aperture at any other $n$ is given by

$$
A(n)=A\left(10^{7}\right) \frac{n^{-0.065}}{0.35}
$$

In what follows, $A$ will stand for $A\left(10^{7}\right)$.

The relationship between the bend and quadrupole coil diameters, and the dynamic aperture, was found to be approximated by:

$$
\begin{aligned}
\frac{1}{A} & =\left(\frac{1}{A_{q}^{2}}+\frac{1}{A_{b}^{2}}\right)^{1 / 2} \\
A_{b, q} & =C\left(\frac{d}{D_{o}}\right)^{m}, D_{o}=40 \mathrm{~mm} \\
A_{b} & =2.7 \mathrm{~mm}\left(\frac{d}{D_{o}}\right)^{3.2} \\
A_{q} & =6.4 \mathrm{~mm}\left(\frac{d}{D_{o}}\right)^{1.9}
\end{aligned}
$$

The aperture as a function of coil diameter, $d$, could be expected to vary approximately as in Table 4 with $10 \sigma=3.8 \mathrm{~mm}$.

Table 4:

\begin{tabular}{cccc} 
Quad Diameter & Bend Diameter & $A(\mathrm{~mm})$ & $A / \sigma$ \\
\hline 4 & 4 & 2.5 & 6.5 \\
4 & 5 & 4.0 & 10.5 \\
5 & 5 & 4.6 & 12.1 \\
4.8 & 4.8 & 4.2 & 11
\end{tabular}

For the basis of comparison here, a simplistic approximate cost model is used to evaluate the relative effectiveness of the incremental coil changes, injection energy changes, cell length changes, etc. It should be stressed tha: these approximate models are not a substitute for detailed cost estimates, but only a guideline. They try to approximately reflect the relative proportion of fixed cost to cost associated with change in diameter. For instance cryostat and labor costs do not necessarly change linearly with coil diameter. Costs associated with change in cell length are discussed below. Only the inpact on the quad has been taken tinto account, although it is known that increasing the number of bend magnets for the same intergial B.dl does increase the cost.

For the bend, quad and correctors, assume the differential cost goes like

$$
\frac{\Delta \cos t}{\cos t}=\frac{3}{4} \frac{\Delta R}{R}
$$


and for the spools, assume

$$
\frac{\Delta \cos t}{\cos t}=\frac{3}{8} \frac{\Delta R}{R} .
$$

Then these assumptions lead us to the results shown in

Relative to a $5 \mathrm{~cm}$ dipole and a $4 \mathrm{~cm}$ quad, the $4.8 \mathrm{~cm}$ dipole and quad would be approximately cost and aperture neutral (with a small aperture benefit). The $4.8 \mathrm{~cm}$ diameter is desirable over the $4 \mathrm{~cm}$ quad because it permits a larger diameter smooth beam pipe with lower impedances. The aperture as a function of injection energy is given in Table 6 for the average of two simulation assumptions.

Table 5 (given in relative technical cost units).

Table 5:

\begin{tabular}{lcc} 
& $\Delta c u / \sigma$ & cu(base line) \\
\hline for bends & 15 & 410 \\
for quad/spool/corrector & 10 & $47+28+09$
\end{tabular}

Table 6:

\begin{tabular}{cccc}
$E_{\text {inj }}(\mathrm{TeV})$ & Aperture & $10 \sigma$ beam $(\mathrm{mm})$ & Aperture $/ \sigma$ \\
\hline 3 & 4.7 & 3.1 & 15 \\
2 & 4.2 & 3.8 & 11 \\
1.8 & 4.0 & 4.0 & 10 \\
1.5 & 3.6 & 4.4 & 8 \\
1.0 & 2.25 & 5.3 & 4.2
\end{tabular}

The result is an almost linear increase in the number of $\sigma$ 's with injection energy, or about $1 \sigma$ per $0.2 \mathrm{TeV}$ of energy increase. Below $1.5 \mathrm{TeV}$ the aperture from simulation results drops rapidly to about 2 to $2.5 \mathrm{~mm}$ at $1 \mathrm{TeV}$. Presumably, this could be understood and compensated to some extent by mid-cell correctors. However, the drift in persistent currents will cause more and more severe problems at lower energy. These are not part of the simulation.

Using $1 \sigma$ per $0.2 \mathrm{TeV}$, a differential cost and figure of merit for increased energy can be estimated. HEB cost, including civil construction and beam lines is about $114 \mathrm{cu}$ for $2 \mathrm{TeV}$ so injection energy gives $11 \mathrm{cu} / \sigma$ aperture increase or decrease.

Injection at $1 \mathrm{TeV}$ with conventional warm magnets has been suggested. An argument being that injection time could be substantially reduced with an 
associated increase in aperture. Ignoring the fact that a $1 \mathrm{TeV}$ conventional ring would not fit on site and the bi-polar injection would be required of a conventional iron magnet system, we can look at some of the other features.

The present $200 \mathrm{GeV}$ MEB has a cycle time of $4.5 \mathrm{sec}$, whereas the HEB has a collider fill time of $72 \mathrm{~min}$. Assuming a $1 \mathrm{TeV}$ MEB has a 4 times longer cycle time and needs 16 cycles to fill the collider, gives $290 \mathrm{sec}$ or about 5 minutes for a reduction factor of .07 compared to the previous filling time. The associated increase in dynamic aperture is $.07^{-.065}=1.19$. However the beam $\sigma$ is $\sqrt{2}$ larger, so Aperture/ $\sigma$ is 0.84 of the previous case. This does not yet take into account the shrinking dynamic aperture with energy to $2.25 \mathrm{~mm}$, resulting in an aperture acceptance of $5 \sigma$ (or $1 / 2$ of our $10 \sigma$ requirement).

The cost of such an injector can be scaled from the MEB, leaving out beam line components but including conventional construction. The cost of the MEB is $\sim 36 \mathrm{cu}$. We would need to scale by 4.5 to about $162 \mathrm{cu}$ for a $1 \mathrm{TeV}$ warm HEB. This is to compare with the HEB cost without beam lines of $108 \mathrm{cu}$. Even if one assumed one didn't need the present MEB, the cost would be about $17 \mathrm{cu}$ more for a $1 \mathrm{TeV}$ instead of a $2 \mathrm{TeV}$ injection complex.

What is at play here is that conventional and superconducting accelerators cost approximately the same per linear foot, with typically superconducting accelerators being $10-20 \%$ more expensive.

In the light of trying to understand the cost optimization of the Collider and to find a cost neutral way to increase the quad/spool aperture to $5 \mathrm{~cm}$, a tabulation of the effects of cell length and phase advance is appropriate. Assuming that about $20 \%$ decrease in integrated quad strength is needed for a cost neutral change, the impact can be evaluated.

If this were taken up by decreasing the phase advance to $71^{\circ}$ but leaving 90 meter spacing, the $\beta$ the spot size $\sigma$ hardly changed. On the other hand, the dispersion function and the dispersion spot size would increase by 1.5 times. The $71^{\circ}$ phase advance has the disadvantage of not canceling dispersion every 2,6 , etc. cells.

If the cell length were changed but $90^{\circ}$ per cell retained, then a $10 \%$ increase in cell length to 100 meters would decrease the quad strength by $10 \%$ and the numbe: of cells by $10 \%$ giving a $20 \%$ net savings. The $\beta$ would be increased by $10 \%$ and the dispersion by $(1.1)^{2}$ or $20 \%$.

At most, the betatron spot has increased $5 \%$ so that if dynamic aperture were unchanged, one would be forfeiting on the order of less than $1 / 2 \sigma$ by this change.

Simulations give a decrease in aperture of about $2 \sigma$ in changing the phase advance from the $1 / 2$ cell length 90 to 70 degrees or in changing from 90 meters to 100 meters.

This reduction would only be partially made up by an increase at the quadrupole aperture to $5 \mathrm{~cm}$. equivalently we are losing on the order of $2 \sigma$ 
of aperture for a savings of about 9 cu's. Moving in the other direction larger phase advance gives no improvement. Shorter $1 / 2$ cell length $(80 \mathrm{~m})$ gives an increase of a little more than $1 \sigma$.

There are a number of trade-offs that could, in principal, be considered in order to accommodate larger quad coil size - e.g., reducing the dipole coil diameter, increasing the cell length, decreasing phase advance or injection energy. Approximate cost per differential spot $\sigma$ can be estimated for the various choices and is displayed in Table 7 .

Neither increased cell length nor reduced phase advance seem to be reasonable alternatives at this point in the project as they disrupt too many other areas of the base line. A similar statement is probably equally true for the Collider bend coil diameter and HEB circumference.

Table 7:

\begin{tabular}{lc} 
& $\Delta \mathrm{cu} / \sigma$ \\
\hline bend coil diameter & $14 \mathrm{cu}$ \\
quad spool coil diameter & $10 \mathrm{cu}$ \\
injection energy & $11 \mathrm{cu}$ \\
phase advance, cell length & $5 \mathrm{cu}$
\end{tabular}

* Loss in effective aperture only

The choice of parameters seems reasonably optimized within the accuracy of the approximation and indicates that, if anything, higher injection energy is more cost effective than coil diameter.

\section{High Energy Booster}

It would be highly desirable if the HEB could use (with minor modification) the $5 \mathrm{~cm}$ magnets developed for the Collider. Simulations of dynamic aperture for the HEB are on-going. The needed aperture for the present $200 \mathrm{MeV}$ injection energy and $38 \mathrm{~m}$ half cell with two $15 \mathrm{~m}$ dipoles is $7.1 \mathrm{~mm}$ for $10 \sigma$ of a $0.8 \pi$ emittance beam. This emittance allows for $0.2 \pi$ dilution in processes associated with HEB and Collider acceleration and transfer. The potential test beam option for the HEB specifies $4 \pi$ emittance $(95 \%$ beam $=4.6 \mathrm{~mm})$. The $10 \sigma$ aperture design is not required for this mode of operation; however, it is clear that much less than $7 \mathrm{~mm}$ aperture might make for unacceptable losses in fixed target operation.

Parameter fits developed for the Collider simulations can be used for a zero order estimate of the HEB aperture. HEB injection time is about $12 \mathrm{sec}$ or $3 \times 10^{5}$ HEB turns. In some approximate sense, one might expect this to be 
equivalent to looking at the Collider aperture for $3.8 \times 10^{4}$ turns:

$$
A_{H E B} \approx A_{C o l l i d e r}\left(@ 10^{7}\right)\left(\frac{n_{H}}{n_{C}}\right)^{-.065}=1.4 A_{C o l l i d e r} .
$$

Using the Collider aperture of $4.6 \mathrm{~mm}$ for $5 \mathrm{~cm}$ quads and bends leads to an expected HEB aperture of $6.4 \mathrm{~mm}$. This is to be compared with an actual HEB simulation at $\Delta p / p=5 \times 10^{-4}$ which gives a result of $5.8 \mathrm{~mm}$ (and $6.1 \mathrm{~mm}$ for $\left.\Delta p / p=7.5 \times 10^{-4}\right)$. Thus we see that the simulated aperture does not comfortably fit the design requirement. However, the required $5 \sigma_{p} / p$ for the HEB is $2.5 \times 10^{-4}$ and the design calls for use of $2 \frac{1}{2} \mu$ filament wire. These effects together sufficiently boost the aperture. Simulation of a $6 \mathrm{~cm}$ coil for the HEB gives $10 \mathrm{~mm}$ of aperture, a substantial increase over the $5 \mathrm{~cm}$ coil. At one time it was felt that this aperture might be necessary as well for cooling to remove eddy current $\mathrm{AC}$ losses from the fast cycling HEB. Further investigation of the cooling indicates it is adequate for $5 \mathrm{~cm}$.

Results of simulations are given in Table $8 \mathrm{a}$. There is a dependance on $\Delta p / p$. The $5 \sigma \Delta p / p$ for baseline Collider operation is $2.5 \times 10^{-4}$. The potential for higher intensity and test beams make it desirable to consider greater $\Delta p / p$ as well, and $7.5 \times 10^{-4}$ has been used in much of the analysis.

Source of the options considered are

1. base line (as function of $\Delta p / p$ )

2. setting average $b_{2}, b_{4}, b_{6}$ to values consistent with $2.5 \mu \mathrm{m}$ filament diameter (as function of $\Delta p / p$ )

3. shorter half-cell length $-32.5 \mathrm{~m}$ instead of $38 \mathrm{~m}$

4. $240 \mathrm{GeV}$ instead of $200 \mathrm{GeV}$ (as function of $\Delta p / p$ ) injection

5. both (as function of $\Delta p / p$ )

6. $6 \mathrm{~cm}$ bend coil diameter

Also included in the calculations are sorting on $b_{2}$ and setting systematic $b_{2}$ $b_{3}, b_{4}, b_{8}$ to zero.

1. setting systematic $b_{2}, b_{3}, b_{4}, b_{6}$ to zero

2. setting systematic $b_{3}$ to zero

Sorting on $b_{2}$ might require cold measurements of all magnets. Setting systematic $b_{2}, b_{4}$, and $b_{6}$ to zero is modeled from the idea that if nickel filament were incorporated in the strand in the proper proportion, it could compensate for the persistent current effects. It could not, however, compensate for the drift in persistent currents. The use of $2.5 \mu \mathrm{m}$ filaments instead of $6 \mu \mathrm{m}$ on which the 
multipole set is based would reduce the systematic persistent current multipoles by $2.5 / 6$ as well as reduce the cooling requirements to about $2 / 3$ of that required for $6 \mu m$ coils.

It is clear that going to a $6 \mathrm{~cm}$ diameter coil completely satisfies the dynamic aperture requirements with comfortable margin and gives about a $5 \sigma$ (about $50 \%$ ) increase in dynamic aperture. Going to a shorter cell or higher energy injection each gain about $10 \%$ to $20 \%(1$ to $2 \sigma)$ and the combination of both yields $20 \%$ to $50 \%$. The improvement is more pronounced at $\Delta p / p=5 \times 10^{-4}$ and below. It is less obvious at $7.5 \times 10^{-4}$.

The "sorting" of $b_{2}$ and the reduction of systematics seem to have a more positive effect $(1 \sigma)$ relative to the base line cell length and less effect when added to the shorter cell simulations. All in all, there is enough scatter in the results to restrain one from putting too fine an interpretation on them.

As in the collider, very approximate cost benefit implications can be attempted. For the coil diameter, using

$$
\frac{\Delta \cos t}{\text { cost }}=\frac{3}{4} \frac{\Delta R}{R}=.15
$$

for the dipole, quad, and correctors/spool gives about $1.7 \mathrm{cu} / \sigma(6 \mathrm{~cm}=$ cost increase of 9.4cu). Actual estimates of the dipole cost indicate $10 \%$ increment is more appropriate.

The cell length cost can be approximated by linear scaling of the total quad/spool integral strength increase of (38/32.5):

$$
\frac{\Delta \cos t}{\cos t}=\frac{3}{4} \frac{\Delta L}{L}+1 \frac{\Delta N}{N} \approx 0.30
$$

where $N$ is the number of components. For the quad/spool correctors, this gives about $3.4 \mathrm{cu} / \sigma$ (increase of $6.2 \mathrm{cu}$ ) assuming about a $2 \sigma$ improvement.

Higher energy injection from the MEB costs about $8.8 \mathrm{cu} / 40 \mathrm{GeV}$ if we scale the total MEB and beam line costs. Again, assuming about a $2 \sigma$ improvement, this gives about $4.2 \mathrm{cu} / \sigma$.

Here, if the magnet field in the MEB could be pushed effectively without an increase in the MEB circumference, then the cost would be substantially less. Operaiion of the MEB above $2 \mathrm{~T}$ (from a nominal of $1.75 \mathrm{~T}$ ) seems unlikely and would only yield an increase of about $25 \mathrm{GeV}$ te $30 \mathrm{GeV}$.

Smaller filament wires, etc., have of the order of $5 \%$ effect on cost of the magnets with a potential cost/benefit of $2.5 \mathrm{cu} \sigma$ if it could be realized.

Sorting on $b_{2}$ might require cold measurements resulting in about $1.4 \mathrm{cu} \sigma$.

A cost/benefit summary (very approximate) is shown in Table 8 . The reader is again warned fo the debateably nature of the cost analysis.

A trade-off which from the above would seem attractive would be to go to the $6 \mathrm{~cm}$ coil and reduce the injection energy by 40 to $50 \mathrm{GeV}$. This would be approximately cost neutral and increase the aperture. Trade off with longer cell 
Table 8: HEB Differentials

$\begin{array}{ll} & \text { cu/ } \sigma \\ \text { coil diameter } & 1.7 \\ \text { cell length } & 3.4 \\ \text { injection energy } & 4.2 \text { (less if no circumference change) } \\ \text { filaments } & 2.5 \\ \text { sorting } & 1.4\end{array}$

length could also be contemplated. In either case, further simulations would be required as the changes would be substantial.

In reality, lower injection energy is not feasible because of the test beams requirement.

A $6 \mathrm{~cm}$ coil for the HEB could be approximately cost neutral if the HEB energy were reduced to $1.8 \mathrm{TeV}$ and if $6 \mu \mathrm{m}$ filament wire were used. This would appear very attractive were it not felt that there is substantial risk in undertaking the development of yet another style of superconducting magnet and for the loss of about $1 \sigma$ of collider aperture.

In summary, the $6 \mathrm{~cm}$ coil has been dropped from consideration. The $5 \mathrm{~cm}$ coil with $2 \frac{1}{2} \mu$ filaments meets requirements and gives about $1 \sigma$ more aperture than a $5 \mathrm{~cm}$ coil with $6 \mu$ filaments. Reducing the half cell length from $38 \mathrm{~m}$ to $32.5 \mathrm{~m}$ increases the aperture about $1.5 \sigma$ over the $2 \frac{1}{2} \mu$ filament, $38 \mathrm{~m}$ case.

The cost of this option of reducing the cell length needs to be looked at in more detail. It is in all likelihood the most practical and needs serious evaluation. Possibly it can be done more economically than indicated and that some savings can be obtained by deciding to use $6 \mu \mathrm{m}$ wire. Here again, there is the feeling that $2.5 \mu \mathrm{m}$ filaments or nickel wire have sufficient development risk so that counting on them as part of the base design may be impractical.

In comparing the cost trade-off numbers for the Collider and HEB, it appears that for the Collider it is more economical to go to higher energy and smaller coil diameter, whereas for the HEB the opposite is indicated. This is probably a reflection of the fact that the Collider is two rings being fed by one superconducting injector. The HEB is only one ring and it is fed by a conventional warm injector which is much more expensive per unit energy.

A final comment about design to cost and optimization of that design. Such optimization can be done only if one is willing to go back and open areas of design which have already been frozen (e.g. Collider coil aperture $-4.8 \mathrm{~mm}$ vs. $5 \mathrm{~mm}$ ) or to take on development risks (e.g. $6 \mathrm{~cm} \mathrm{HEB}$ ). The former process is extremely destructive to forward progress on the project and probably is not cost effective. The latter is a balancing of risk and schedule vs. potential long-term benefit. 


\section{The LEB Peak Energy, Ring Circumference and the MEB Injection Energy}

The choice of LEB circumference (and injection energy) determines the space charge tune shift of that accelerator and sets the brightness scale of the whole accelerator chain. Thus, the LEB circumference is a parameter which must undergo considerable technical scrutiny through cost effectiveness is not an important criterion. In looking at this parameter it is important to keep in mind that the $\Delta \nu, c$ scales linearly with radius $(\mathrm{R})$ and that small reduction is in $\mathrm{R}$ at the penalty of forcing marginal space allocation for engineering equipment (e.g. If systems, magnet ends etc.) is not prudent. Big reduction like a charge in radius to $2 / 3$ its present value begins to have significant impact. Of equal importance is the consideration that the LEB injection energy is upgradeable and tunnel space is provided between the Linac and LEB for a linac upgrade to $1 \mathrm{Gev}$ cup (KE) from $600 \mathrm{MeV}$. Alternatively or a small prebooster could be added at a later time. The $1 \mathrm{Gev}$ linac reduces LEB space charge by a factor of 1.75. A prebooster could in principal reduce it even more.

In setting LEB to MEB parameters it should be assured that the MEB will not become the performance bottleneck of the accelerator chain. In the LEB, peak energy, fast cycling $(10 \mathrm{~Hz}$ resonant excitation) and avoidance of transition crossing are factors. In the MEB space charge tune shift, emittance dilution from beam-gas multiple scattering and injection field quality must be considered. (The latter is not considered to be a limitation within any feasible range of injection energy.)

In both the LEB and MEB, transverse emittance growth is the parameter that is driving the design choices, and our understanding of the process and what is important is limited. Experience from operating accelerators is hard to extrapolate because of the small emittance and growth required. In particular the emittance growth with space charge tune shift as a function of dwell time at injection is not completely understood (at least by us). A fast cycling LEB has been chosen to minimize the time spent at injection in both the LEB and MEB and to minimize the growth from space charges as well as gas scattering (MEB) (This choice also allows for reasonable test beam fixed target capability as well).

The $10 \mathrm{~Hz}$ operation needs sufficient space for if cavities. Transition has been avoided in the LEB because of the experience with the FNAL booster and longitudinal instabilities observed there. It is not known to what extent these might also effect transverse emittance but it is recognized that precise beam manipulations at transition can be tricky in a fast cycling accelerator. The avoidance of transition in the LEB makes for a non conventional lattice and possibly not as efficient utilization of space as might otherwise be achieved.

Table $9 \mathrm{a}$ - tabulates the approximate circumference penalty associated with 
fast cycling, transition, and the difference in peak momentum of 10 to $12 \mathrm{GeV} / \mathrm{c}$.

Table 9: Tune Shifts LEB and MEB as Function of LEB Circumference and Energy

\begin{tabular}{|c|c|c|c|}
\hline LEB Circumference/p & LEB & LEB/1.75 & MEB Inj./1.75 \\
\hline $570 / 12 \mathrm{Gev} / \mathrm{c}$ & 0.6 & 0.34 & $0.08 / 014$ \\
\hline $475 / 10 \mathrm{Gev} / \mathrm{c}$ & 0.5 & 0.28 & $0.11 / 0.20$ \\
\hline $380 / 8 \mathrm{Gev} / \mathrm{c}$ & 0.4 & 0.23 & $0.18 / 0.31$ \\
\hline \multicolumn{4}{|c}{ Note $12 / 3 \quad 4 / 5$} \\
\hline
\end{tabular}

1) Nominal intensity, $0.6 \mathrm{GeV}$ or 1.75 nominal at $1 \mathrm{GeV}$

2) Nominal intensity $1.0 \mathrm{GeV}$ LEB

3) Nominal intensity MEB at injection

4) 1.75 brightness MEB - at injection.

In the LEB space charge the shift is estimated to be 0.6 at $0.4 \pi \mathrm{mm}-$ mrad emittance and $600 \mathrm{Gev}(\mathrm{KE})$ injection. This estimate is controversial as it depends strongly on the bunching factor assumed. The typical range in $\Delta \nu$ is 0.4 to 0.6 consistent analysis leads to the same sort of value for the FNAL Booster operation. This is reduced by 1.75 at one $\mathrm{GeV}(\mathrm{KE})$.

The MEB with $12 \mathrm{Gev} / \mathrm{c}$ injection has a space change tune shift of 0.08 during the injection dwell. This will increase during the start of ramps to approximately 0.12 depending on ramp conditions. These numbers appear reasonably small but still may be difficult to fit within major resonant lines. Low energy dwell times are fractions of a second, not msecs as in the LEB.

With an LEB upgrade to increased $N / \epsilon$ by 1.75 the MEB numbers for injection becomes 0.14 . One can then ask what happens at lower injection energy (linearly smaller radius LEB) to the tune shifts in both rings. (Table 9b) What is at play here is that the LEB tune shift gets smaller linearly with energy (R) and the MEB tune shift increases inverely with the energy squared. At $8 \mathrm{Gev} / \mathrm{c}$ the $\Delta \nu$ is almost equal in the two rings under the condition of upgraded linac energy (0.4 to 0.31 ). Considering the relative dwell time of the two accelerators this would seem to be an optimum condition.

Emittance growth in the MEB from beam gas multiple scattering goes like $d \epsilon \propto \frac{P t}{\gamma}$, where $P$ is pressure $\left(5 \times 10^{-8}\right.$ nominal $)$ and $t$ is dwell time.

For the nominal design $\left(12 \mathrm{Gev} / \mathrm{c}, 5 \times 10^{-8}\right.$ torr, $1 / 2 \mathrm{sec}$ injection time $)$ this gives $\Delta \varepsilon=0.077 \mathrm{~mm}-\mathrm{mr}$ or $7 \%$ of the emittance growth budget for the MEB of $0.1 \mathrm{~mm}-\mathrm{mr}$. This is well within a possible allotment of $0.02 \mathrm{~mm}-\mathrm{mmr}$ for this contribution. Injection energy is not a serious limitation here. However a decrease in LEB cycletime to say $1 \mathrm{~Hz}$ would require careful investigation of the 
MEB achievable vacuum. The criteria to use would be beam pipe bake before magnet assembly but without insituo bake. Experience would indicate factors of at least 2 below $5 \times 10^{-8}$ may be achievable. Larger factors have been reported serious investigation would be necessary before changing the nominal pressure to be a lower value. .

The choices of LEB energy, cycle rate, transition gamma, lattice and resulting circumference have been made largely on the basis of experience. The allowance for an LEB injection energy increase is a fundamental part of this choice. A smaller circumference $10 \mathrm{Gev}$ LEB is potentially as satisfactory a choice, and may need to be revisited if significant circumference redution could be realized (ie $\sim 475 \mathrm{~m}$ )

\section{Medium Energy Booster}

\subsection{Choice of Peak Momentum}

During the site specific design development, the choice of $200 \mathrm{Gev} / \mathrm{c}$ for the MEB was somewhat arbitary and was based on the need to raise the injection energy into the now 2Tev HEB and on the requirements of the test beams for highest possible lepton energies. The $200 \mathrm{Gev} / \mathrm{c}$ MEB was part of the June 1990 cost estimate.

Since the review, analysis of the HEB injection aperture has undergone optimigation studies. One solution purosed was to further increase the MEB peak momentum. Two approaches to higher momentum in the MEB are herein addressed. The first is to increase the circumference. This solution in technically sound. The only anticipated implication on machine performance is that of space charge tune shift at injection. This issue will be discussed later with respect to the arguments of the choice of injection parameters. The increased cost of the MEB is expected to scale with the increase in circumference, and therefore with the final momentum. Thus the machine would cost:

$36 c u \times(P / 200)$

where $P$ is the choice of top momentum. It is to be noted that considerable work has been done in siting the injector machines, and civil construction design work is proceeding on the linac. Therefore, the position has been taken that a change to the circumference of the MEB is not an option.

The second approach to higher momentum in the MEB is to increase the strength of all magnetic elements. In addressing this idea, it should first be noted that there is no excess room in the MEB lattice to make devices stronger by increasing their length. The MEB at a peak momentum of $200 \mathrm{GeV} / \mathrm{c}$ and 
a circumference of 3960 meters is tight for free space. All the drift spaces have been made as short as possible. The space for high momentum extraction is 25 meters. A shorter straight region would complicate extraction. Furthermore, three high momentum extraction locations are needed, and to retain symmetry the ring has six. Space has been retained in the lattice for the possible inclusion of $\gamma_{t}$ jump quadrupoles, and the necessary inclusion of slow extraction devices.

The only other way to increase the strength of the elements is to increase their magnetic strength. Clearly this cannot be done without limit. The magnetic strength of the main dipoles is $1.75 \mathrm{~T}$ and that of the quadrupoles is $23.8 \mathrm{~T} / \mathrm{m}$ at $200 \mathrm{GeV}$; the strength scales directly with momentum.

The chromaticity sextupoles, which are already strong devices, will also have to become somewhat stronger. It is not possible to quantify the increase since it is dependent on the saturation characteristics of the dipole, and at present this information is not known. If the quadrupoles are made appreciably stronger, they will require more iron and a larger profile than presently specified. This will introduce a complexity into the extraction system since the departing beamline will first have to avoid a quadrupole.

\subsection{Choice of Injection Momentum}

The present choice for the injection momentum is $12 \mathrm{GeV} / \mathrm{c}$. Any motivation for a change to this parameter is from the LEB, in an attempt to alleviate problems there. Arguments in behalf of the MEB to retain the $12 \mathrm{GeV} / \mathrm{c}$ parameter must address technical issues associated with the ability of the MEB to meet its design requirements. Specifically, this choice is not too dependent on cost issues, but really rests on technical arguments. Clearly, from an MEB point of view, higher injection momentum are better. Therefore each issue must be questioned based on what risks are incurred by lowering the injection momentum below 12 $\mathrm{GeV} / \mathrm{c}$. The following are the issues.

\subsubsection{Emittance growth due to beam gas scattering}

The model for emittance growth is: $[8,9]$

$$
<d \epsilon / d t>/ \pi=\text { constant } \times \text { vacuum pressure } / \gamma .
$$

For the MEB at $12 \mathrm{GeV} / \mathrm{c}$, and at the design vacuum pressure of $5 \times 10^{-8}$ torr, this is

$$
<d \epsilon / d t>/ \pi=.0143 \mathrm{~mm}-\mathrm{mrad} / \mathrm{sec} \text {. }
$$

The MEB dwell time for the first LEB batch is about .5 sec., so that it will experience growth of $0.0072 \mathrm{~mm}-\mathrm{mrad}$. This is $7 \%$ of the MEB emittance growth budget, and is not considered a problem. A reduction in the MEB injection momentum to $10 \mathrm{GeV} / \mathrm{c}$ would increase this to $9 \%$. This also is not a problem. 
Note however that a reduction of the LEB cycle time by a factor of 10 (as has been suggested) would have an impact, and lead to a more stringent vacuum requirement in the $\mathrm{MEB}$.

\subsubsection{Transverse emittance growth due to space charge tune shift}

It is a straight forward calculation to derive the space charge tune shift. It is more difficult to determine the subsequent emittance growth. With the standard collider parameters, the calculated space charge tune shift at injection is 0.077 . This is consistent with what has been achieved in other machines in the momentum range of the MEB. Simulation deriving emittance growth from space charge tune shift are still focusing on the LEB problem. Results for the MEB are anticipated during the summer of 1991. Simply lowering the injection momentum to $10 \mathrm{GeV} / \mathrm{c}$, while leaving other parameters the same, would increase the space charge tune shift parameter to .11 . Of all the arguments supporting the $12 \mathrm{GeV} / \mathrm{c}$ injection momentum, this is the strongest. However, as pointed out above, the real problem, emittance growth cannot be strongly tied to the tune shift parameter, so no claim can be made, as of yet, as to what injection momentum is acceptable, and what is not.

\subsubsection{Dynamic Aperture}

The dynamic aperture varies as a function of injection momentum through two mechanisms. Firstly, the beam size goes as the inverse of the square root of gamma. Secondly, the field quality deteriorates as the injection momentum goes down. The present design of the MEB anticipates a good dynamic aperture at $12 \mathrm{GeV} / \mathrm{c}$. While no explicit MEB tracking has yet been done, tracking studies performed on the Fermilab Main Injector indicate a dynamic aperture of about $17.5 \mathrm{~mm}$. (these tracking studies were done independent of beam momentum, that is, the inverse square root of momentum was not yet applied to these results, and the field level at injection to the $\mathrm{MI}$ is the same as the field level of the MEB at injection although the momenta are different.) This corresponds to 10 sigma for collider operation and 4 sigma for test beam operation. If we injection momentum of the MEB was to be lowered to $10 \mathrm{GeV} / \mathrm{c}$, the sigma numbers would become 9 and 3.6 respectively, just from beam size considerations. The question of magnetic field quality, and its impact, is harder to assess. Field nonlinearity, arising from remanent effects is not easy to calculate. Limits on multipoles can be specified so that the sigma parameters are essentially uneffected over the injection momenta range of $10 \mathrm{GeV} / \mathrm{c}$ to $12 \mathrm{GeV} / \mathrm{c}$. However the results will ultimately depend on the quality of the steel that is obtained. These specs are being written very carefully. The argument then, is that the steel will be good enough so that it won't matter to the field quality what the injection momentum is if it in the range of $10 \mathrm{GeV} / \mathrm{c}$ to $12 \mathrm{GeV} / \mathrm{c}$ is used. However, in the absence 
of specific numbers, the higher field is preferable.

The conclusions with respect to the injection momentum is that $12 \mathrm{GeV} / \mathrm{c}$ is preferred by the MEB. All of the arguments are soft, the strongest being that related to space charge. The MEB specs will be written in such a way that operation at $10 \mathrm{GeV} / \mathrm{c}$ is possible if needed as a fall back by LEB limitations.

\subsection{Transition Crossing}

The arguments for choosing a lattice which crosses transition have been given before, and basically involve complexity, an increase in needed circumference, and cost. Furthermore, at the time decisions were being made, a proposal for crossing transition, using a scheme in which the non-wanted longitudinal focusing forces are removed, was suggested. It is this scheme that we are planning on using. Simulations are being done here, and at Fermilab. Again, the main concern for the MEB is that the tight bunching, which normally occurs as transition is encountered, will produce transverse emittance growth. The varying factor in the space charge tune shift equation (the ratio of bunching factor to $\left.1 / \gamma^{*} 2\right)$ is seen in the simulations to be the same at the onset of the non-focusing scheme as it is at injection, and it decreases after that. This scheme continues to receive study. It is noted that there are engineering complexities in the rf system associated with the amplitude and phase control of additional cavities operating at harmonic frequencies. As a contingency, space has been reserved in the lattice, at locations of focusing quadrupoles, for $\gamma_{t}$ jump quadrupoles.

\section{Low Energy Booster}

\subsection{Introduction}

The Low Energy Booster (LEB) is to be the first synchrotron in the injection system. The general design specifications for the LEB are given in Table 10 and 11. Detail information is provided in $[11,12,13,14,15]$

The basic lattice design constraints were a small circumference to minimize space charge effects, adequate azimuthal space for the required hardware, and sufficiently large $\gamma_{t}$ to avoid crossing transition and to provide an adequate slip factor at extraction to support cogging procedures.

Several alternative lattices were explored in light of these specifications and those given in Table 10. A more detailed discussion of the design philosophy and concepts involved in these considerations and tracking evaluations are given in $[13,14]$. The basic lattice parameters are given in Table 11. 
Table 10: LEB Design Parameters

\begin{tabular}{|c|c|}
\hline Parameter & Value \\
\hline Injection Momentum (GeV/c) & 1.22 \\
Extraction Momentum $(\mathrm{GeV} / \mathrm{c})$ & 12.0 \\
Repetition Rate $(\mathrm{Hz})$ & 10 \\
Circumference $(\mathrm{m})$ & 570 \\
Harmonic Number & 114 \\
$\epsilon_{\text {rms }}^{*}(\pi$ mm-mrad) & 0.6 \\
Particles/bunch & $1 \times 10^{10}$ \\
\hline
\end{tabular}

Table 11: LEB Lattice Parameters

\begin{tabular}{|c|c|c|}
\hline & Nominal & \\
Parameter & Value & Range \\
\hline$\nu_{x}$ & 11.65 & $10.85-11.85$ \\
$\nu_{y}$ & 11.60 & $10.8-11.8$ \\
$\chi_{x}$ (natural/corrected) & $-15.7 / 0$ & $-14.0--16.5 / 0$ \\
$\chi_{y}$ (natural/corrected) & $-17.7 / 0$ & $-15.0--18.5 / 0$ \\
$\gamma_{t}$ & 22.1 & $24.4-21.9$ \\
\hline
\end{tabular}

Although the beam intensity requirement for the LEB is relatively modest compared to existing machines, the required small beam emittance results in a significant tune depression. In terms of the Laslett tune shift, the tune depression is $\approx 0.6$ at an injection momentum of $1.22 \mathrm{GeV} / \mathrm{c}$. Therefore, the effects of space charge are considered a major constraint with respect to the beam dynamics. Certainly a smaller space charge induced tune shift is preferable. However, the maximum allowable tune shift is not a well defined number. Experimental results of existing machines indicate that a tune shift of even more than 0.5 may be acceptable. Machine parameters such as resonance strengths, superperiodicity, or operating transverse and synchrotron tunes all have a bearing on the resultant acceptable tune depression.

To explore the relevant parameter space, a code continues to be developed which employs multi-particle tracking with the transverse dynamics of the thin lens code TEAPOT, the space charge force introduced as kicks. Synchrotron oscillations and acceleration are included. Emittance evolution as well as the particle distribution are simulated as a function of time.[15]. 


\subsection{Parameter Evaluations}

The LEB basic design parameters were chosen after significant considerations as explained below. In all cases, technical performance as opposed to cost was the overriding issue commensurate with the total cost of the LEB being only $1 / 2 \%$ of the project cost.

\subsubsection{Injection Momentum}

The baseline injection momentum $(1.22 \mathrm{GeV} / \mathrm{c})$ was chosen to be compatible with FNAL Booster experimental data which imply a maximum acceptable tune shift value of $\approx 0.6$ being a threshold below which there is little emittance dilution. However, because the FNAL data would be statistically consistent with a significantly lower threshold tune shift, because the simple scaling implied by the Laslett tune shift formula may not be sufficiently valid in light of the small LEB emittance growth budget $\left(\Delta \epsilon_{\text {total }} \approx 0.2 \pi \mathrm{mm} \mathrm{mrad}\right)$, and because higher intensities may be desirable in the future, the Linac design is compatible with an increase in the injection momentum to $1.7 \mathrm{GeV} / \mathrm{c}$ which provides (assuming Laslett scaling) a tune shift reduction to $\Delta \nu=0.34$ for the same emittance and intensity conditions. The rf frequency range $(47.5-59.8 \mathrm{MHz})$ necessary to support an injection momentum of $1.22 \mathrm{Gev} / \mathrm{c}$ has been accomplished at FNAL and is therefore, achievable.

\subsubsection{Extraction Momentum}

The extraction momentum of $12 \mathrm{GeV} / \mathrm{c}$ was chosen primarily on technical MEB performance issues. Based upon detailed lattice hardware evaluations, it was found that an extraction momentum of $12 \mathrm{GeV} / \mathrm{c}$ required a circumference of $570 \mathrm{~m}$ and that an extraction momentum of $10 \mathrm{GeV} / \mathrm{c}$ required a circumference of $540 \mathrm{~m}$ If a smaller circumference could be realized then $10 \mathrm{Gev} / \mathrm{c}$ would become desireable. .

In favor of a $540 \mathrm{~m}, 10 \mathrm{GeV} / \mathrm{c}$ LEB were a reduction in cost due to reduced integral dipole length $(\approx 17 \%)$, and reduced $\mathrm{RF}$ voltage requirement $(\approx 15 \%)$.

Against a $540 \mathrm{~m}, 10 \mathrm{GeV} / \mathrm{c}$ LEB with respect to the LEB performance was the potential increase in space charge effects $(\approx 12 \%)$ due to a lower average ramp rate. The MEB would have, with the lower LEB extraction momentum, a potential decrease in dynamic aperture due to remnant fields, an increase in the space charge tune shift $(\approx 45 \%)$, and an increase in emittance dilution due to multiple scattering from residual gas $(\approx 20 \%)$. In addition, there would be a larger frequency range $(\approx 45 \%)$ required of the MEB rf system.

In conclusion, it was felt that the overall system (LEB \& MEB) performance would be enhanced with a higher design value for the LEB extraction momentum more than offsetting the possible slight cost savings due to the reduced rf and dipole requirements. This solution also provides a technical contingency. 


\subsubsection{Lattice Design}

The choice of lattice designs revolved primarily around the issue of $\gamma_{t}$. The value of $\gamma_{t}$ was chosen to avoid crossing transition, i.e., $\gamma_{t} \geq 12.8$. In a fast cycling machine $(10 \mathrm{~Hz})$, where the transverse emittance (at intensity) is the single most important parameter, the penalty of crossing transition was felt to be unnecessary since it could be avoided by appropriate choice of lattice. Although schemes exist, in principle, to mitigate the bunch compression at transition; to assume that they could adequately be implemented in a fast cycling machine represents a significant technical risk. In lieu of these schemes, emittance dilution of some magnitude is unavoidable.

A further criterion with respect to $\gamma_{t}$ was to have an adequate value of the slip factor [define] at extraction to support possible LEB to MEB cogging scenarios. Based upon these analyses a $\gamma_{t} \geq 20$ was found to be desirable. A standard FODO lattice results in a value of $\gamma_{t} \approx \nu$. The SCDR LEB design utilized a high tune to achieve a value of $\gamma_{t} \approx 14.5$ with a circumference of $540 \mathrm{~m}$. To expand the SCDR concept to a value of $\gamma_{t} \approx 20$ would have required a significant increase in circumference. (The SCDR design could not have been constructed within a circumference of $540 \mathrm{~m}$ because of insufficient free space allocation).

However by creating an oscillation around the ring of the dispersion function, the the value of $\gamma_{t}$ is increased significantly above the tune. Two such designs were evaluated and found to have adequate dynamic aperture and acceptable sensitivity with respect to perturbations (mispowering, multipoles, and misalignment).

Therefore, since a lattice design exists which provides the appropriate values of $\gamma_{t}$ and circumference, and has adequate dynamic aperture and acceptable error sensity, it has been adopted as the reference design.

\subsubsection{Ramp Rate}

Several possible acceleration ramp profiles were explored. These included both resonant and linear systems. The resonant power system cost increased as the frequency decreased with the inverse true of the linear system. The cost maximum of both was at $\approx 5 \mathrm{~Hz}$. The required $\mathrm{rf}$, and therefore the cost scale with frequency.

Because the effects of space charge are likely to be exacerbated by a slower cycle rate, because the emittance dilution due to gas scattering in the MEB would be increased (or require an improved and more costly vacuum system) with slower cycle rate, and because it is always possible to run a machine at a slower rate than the design value, the $10 \mathrm{~Hz}$ resonant cycle was chosen.

In order to provide improved diagnostic capability for commissioning, and to have the option of a linear ramp, the circuit chosen allows, at the expense

of several hours of labor, the ability to run the LEB with a linear ramp. The 
initial power supplies required for the $10 \mathrm{~Hz}$ system are compatible with a $0.3 \mathrm{~Hz}$ linear ramp. Implementation of additional power supplies allows the possibility of higher linear ramp rates.

The magnets were specified compatible with the $10 \mathrm{~Hz}$ ramp rate. This resulted in a peak field value of $1.3 \mathrm{~T}$ for the dipoles and a maximum gradient of $14.9 \mathrm{~T} / \mathrm{m}$ for the quadrupoles. The dipoles and quadrupoles will be in a single resonant circuit. The laminations of the dipole and quadrupole have been carefully designed to ensure that there is good tracking between them during the ramp, and trim quadrupoles have been specified to provide a full integer tune variation, to correct for tracking errors, and to reduce the half-integer resonance stop band.

\section{$7 \quad$ Linac}

The linac injector for the LEB provides a $600 \mathrm{MeV}, 25 \mathrm{~mA} \mathrm{H}^{-}$beam at a $10 \mathrm{~Hz}$ pulse rate with normalized rms transverse emittances $<0.4 \pi \mathrm{mm}$-mrad in both planes. The pulse length is $\sim 8 \mu \mathrm{sec}$ for three-turn injection of $\sim 10^{12}$ protons into the LEB in Collider-fill mode, and $\sim 35 \mu \mathrm{sec}$ for 16-turn injection of $5 \times 10^{12}$ protons in test-beam mode. The design of the linac is conventional, beginning with a $35 \mathrm{kV}$ ion source and LEBT, followed by an RFQ in which the energy is increased to $2.5 \mathrm{MeV}$. The $2.2 \mathrm{~m}$ long RFQ operates at $427.6 \mathrm{MHz}$, and has peak surface fields of $36 \mathrm{MV} / \mathrm{m}$, which corresponds to 1.8 times the Kilpatrick "limit." Improved performance is achieved by increasing the vane voltage slightly as the particle energy is increased.

The RFQ is followed by a drift-tube linac, also operating at $427.6 \mathrm{MHz}$, which increases the proton energy to $70 \mathrm{MeV}$. Higher output energies were considered, but the desire to minimize costs provided an incentive to switch to the less expensive coupled-cavity structure as early as possible. Peak surface fields in the DTL are held to $28 \mathrm{MV} / \mathrm{m}$, or 1.4 times the Kilpatrick limit. The reduction in field, relative to the RFQ, is held to be necessary in view of the $24 \mathrm{~m}$ length of the DTL, so that the relative probability of sparking is nearly equal in the two devices. The 4-tank DTL will use permanent magnet quadrupole 3 in a FODO array, and post stabilizers. Each tank will have its own $4 \mathrm{MW}$ klystron.

A side-coupled linac, operating at $1282.2 \mathrm{MHz}$, the third harmonic of the RFQ/DTL frequency, complete the acceleration to $600 \mathrm{MeV}$. This portion consists of 60 coupled-cavity sections, each with 20 or 22 accelerating cavities, connected into 10 modules of 6 sections each. The sections in each module are connected with bridge couplers, and each module is powered by a $20 \mathrm{MW}$ klystron. The CCL portion of the linac is $111.6 \mathrm{~m}$ long, and the peak surface field of $32 \mathrm{MV} / \mathrm{m}$ is equal to the Kilpatrick limit at this frequency to ensure reliable spark-free operation of this long system.

The linac operating frequencies were chosen to be the 9-th harmonic of the 
LEB if injection frequency. Thus, the option for bunch-to-bucket filling, or "painting" is preserved, even though initial operation scenarios assume the use of adiabatic capture. No sufficiently persuasive case has been made to date that painting schemes can contribute to reduced space-charge tune spread in the LEB, without leading to unacceptably large transverse emittances. In addition, adiabatic capture is a process which is well established and understood.

The LEB design is still troubled with the effects of space charge at injection. In anticipation of this, space has been reserved in the linac building designs for an upgrade of the energy to $1000 \mathrm{MeV}$, simply by installing additional CCL modules. Thus, the decision to upgrade the linac energy and improve operation of the LEB can be taken at any time. The additional costs associated with this upgrade are estimated to be $\sim \$ 15 \mathrm{M}$.

\section{Summary and Conclusions}

Major parameters of the SSC injection chain have been finalized through minor adjustments can still be encorporated if sufficiently justified. An attempt has been made in this paper to describe the complicated interplay of parameters and the various trade-off considerations that enter into parameter choices of the SSC. Particular focus has been given to the series of accelerators through injection to the Collider. In some areas, such as LEB energy, technical uncertainties drive the choice of parameters. Other areas lend themselves in principal to cost optimization. Realistically, however, in a project such optimization often cannot be carried out as parameters which have already been frozen should not be reopened if progress is to be made. A study such as this can support the position that choices already made are rational, but is unlikely to lead to fundamental change unless specific single changes can take place at some cost penality.

\section{Acknowledgements}

In assembling this material, we are playing the roles of rapporteurs; the generation of text has been done by others (who may also be playing the same role, of course). For this paper, our principal sources of words have been Helen Edwards and Bob Palmer of the Project Management Office, and our associates Alex Chao, Warren Funk, Rod Gerig, Dave Johnson, Ken Kauffmann, and Richard York.

\section{References}

[1] "Conceptual Design of the Superconducting Super Collider," ed. J. D. Jackson, SSC Report SSC-SR-2020, Berkeley, March 1986. 
[2] R. Gerig, et al, "Simulations of the Fermilab Main Ring," Proc. of 1987 IEEE PAC, No. 87CH2387-9, March, 1987.

[3] R. Gerig, et al., "Improvements to the Fermilab Main Ring," European Accelerator Conference, Rome, 1988.

[4] D. Edwards and M. Syphers, "Selection of Basic Parameters for the Collider Rings," SSC Report SSCL-297, Dallas, June 1990.

[5] T. Garavaglia, K. Kauffman, and R. Stiening, SSCL-265, SSCLN-739.

[6] D. Ritson, SSCL-305.

[7] R. Palmer, K. Kauffman, private communication.

[8] R. Gerig, "Main Ring Dynamic Aperture Studies," FNAL internal report EXP-164, January, 1989.

[9] M. Syphers, "Beam-Gas Scattering Lifetimes in the Fermilab Main Ring," FN-484, Fermilab, 1988.

[10] R.C. York, et al. "The Superconducting Super Collider Low Energy Booster: A Status Report ", Proceedings IEEE PAC - 1991

[11] N.K. Mahale, et al. "Debunching and Capture Effects in the SSC Low Energy Booster", Proceedings IEEE PAC - 1991

[12] N.K. Mahale, et al. "Longitudinal Matching between LEB and MEB for the SSC", Proceedings IEEE PAC - 1991

[13] E.D. Courant et al. "Low Momentum Compaction Lattice Study for the SSC Low Energy Booster", Proceedings IEEE PAC - 1991

[14] F. Pilat, et al. "Dynamic Aperture and Performance of the SSC Low Energy Booster Lattice ", Proceedings IEEE PAC - 1991.

[15] S. Machida, et al. "Space Charge Effects in the SSC Low Energy Booster", Procoedings IEEE PAC - 1991. 